\title{
Using origami and CAD as tools for spatial ability training for first-year female engineering students
}

\section{Ing. Mayari Illarij Serrano Anazco, Purdue Polytechnic Institute}

MAYARI SERRANO is currently a graduate research assistant in the College of Engineering at Purdue University. She earned her B.S. degree in Biotechnology Engineering from the Army Polytechnic School, Quito, Ecuador. She completed her M.S. in Computer and Information Technology at Purdue University. Mayari is currently a PhD student at Purdue University and is working in for the Women in Engineering Program. Her interests include foster STEM enthusiasm, and technology innovation.

\section{Dr. Suzanne Zurn-Birkhimer, Purdue University-Main Campus, West Lafayette (College of Engineering)}

Dr. Suzanne Zurn-Birkhimer is Associate Director of the Women in Engineering Program and Associate Professor (by courtesy) in the Department of Earth, Atmospheric, and Planetary Sciences at Purdue University. Dr. Zurn-Birkhimer conducts research and leads retention activities including administration of the undergraduate and graduate mentoring programs and the teaching of the Women in Engineering seminar courses. For the past decade, Dr. Zurn-Birkhimer's research has focused on broadening participation of women and underrepresented group in STEM fields. Recently, she has been investigating the intersection of education and career path with cultural identity and is developing strategies to inform programming and policies that facilitate recruitment and retention of underrepresented populations in academia. In 2012 Dr. Zurn-Birkhimer was presented with an Outstanding Alumni Award from the Department of Earth, Atmospheric, and Planetary Sciences and in 2019 the College of Science Distinguished Alumni Award at Purdue University. Dr. Zurn-Birkhimer earned her B.S. in Mathematics from the University of Minnesota, and an M.S. and Ph.D. in Atmospheric Science from Purdue University. 


\title{
Using origami and CAD as tools for spatial ability training for first-year female engineering students
}

\begin{abstract}
This Complete Evidence-based Practice paper will describe the development, implementation, and analysis of an online Spatial Skills Training Workshop employed in a Women in Engineering first-year seminar course over the course of two years.

The ability to mentally manipulate objects is known as spatial reasoning, and those with strong skills in this area have a propensity to pursue and persist in science, technology, engineering, and mathematics (STEM) careers. However, it is widely known that females test lower on spatial reasoning tests than their male peers yet years of research show that strong spatial visualization skills are linked to success in engineering. Perhaps this partially explains the slow growth of females in undergraduate engineering programs over the past two decades.

This research aimed to develop robust and straightforward training modules for female students to improve their spatial abilities so that they do not lag behind their male counterparts as they pursue undergraduate engineering degrees. To that end four 12-week spatial skills training workshops have been developed and implemented into a first-year female engineering seminar course. Weekly modules focused on origami or computer-aided design (CAD) tasks, and were designed to be completed, on average, in one hour.

The goal of this study was to determine if the use of origami and/or CAD positively affected participants' spatial reasoning and, if so, which origami/CAD combination resulted in a greater improvement in skills. The Purdue Spatial Visualization Tests (PSVT) were used to assess spatial aptitude. This study examined the change in PSVT scores before, during, and after completion of the workshop modules. These scores were also evaluated in the context of the participants' country of origin, prior origami and/or CAD experience, as well as whether participants' parents or guardians are engineers.
\end{abstract}

\section{Introduction and Related Work}

The mental steps for representing, analyzing, and outlining inferences from spatial relations are called spatial reasoning ${ }^{1}$. Previous research shows that well-developed spatial skills have a significant correlation to higher overall grades ${ }^{2}$, better science, technology, engineering, and 
mathematics (STEM) skills ${ }^{3}$, higher retention rates in STEM majors ${ }^{4}$, and are indicative of a propensity for $\mathrm{STEM}^{5,6}$.

Gender differences on spatial skills performance have been well-documented. Research suggests that women consistently and significantly score lower than men on spatial skills tests ${ }^{2,7,8,9}$. This disparity is commonly attributed to a combination of environmental factors, and the types of courses, toys, and sports in which a child engages ${ }^{4}$. It is important, then, for women to have the opportunity to improve their spatial abilities early in their college careers as research shows that improving female students' spatial skills would potentially increase retention rates for women in STEM fields ${ }^{2}$.

Fortunately, spatial skills are malleable and research shows that even a small amount of training can have a positive impact ${ }^{10}$. Origami, the Japanese art of paper folding, for example, has been proven to increase spatial visualization skills and level of geometric understanding in middle school students ${ }^{11}$ and spatial visualization and orientation in elementary school students ${ }^{12}$.

Utilizing computer-aided design (CAD) software has also been shown to improve differential aptitude, mental rotation, and spatial visualization ${ }^{13}$ as well as spatial developments, rotations, and views $^{14}$ in middle school students. Similarly, Sorby ${ }^{2}$ states that sketching $3 \mathrm{D}$ drawings has a significant influence in the development of spatial skills.

Hsi et al. ${ }^{15}$ also found that a one-day workshop on 2D and 3D sketching led to an enhancement in spatial strategies to solve engineering problems. A pilot study was conducted by Zurn-Birkhimer et al. ${ }^{16}$ for the development and implementation of a semester-long, 12-module workshop consisting of four origami-based modules followed by eight CAD-based modules ${ }^{16}$. Both the experimental group and the control group saw a statistically significant increase in their spatial ability as determined by the Purdue Spatial Visualization Tests (PSVT). No statistically significant difference was seen in the gains of the experimental group versus gains in the control group however, the experimental group achieved passing test scores of $70 \%$ or greater on each of the three PSVTs by the post-test whereas the control group failed to reach this level ${ }^{16}$.

This study focused on developing an efficient origami/CAD workshop for students to improve their spatial skills outside of the classroom. Engineering curriculum is already very rigid and it is nearly impossible to add an additional required course into the freshman year. Online spatial training has been shown to improve spatial skills over the course of several weeks ${ }^{17}$, and after as little as six contact hours ${ }^{18}$. Therefore, creating an independent, easy to access online workshop could benefit any student wishing to improve their spatial skills or any instructor wishing to add a course component without surrendering in-class instruction time.

\section{Spatial Reasoning Workshops}

The spatial reasoning workshops developed for this study consisted of 12 one-hour modules with the intent to provide participants the opportunity to improve their spatial abilities: spatial perception, mental rotation, and spatial visualization. Modules were intentionally designed with tasks that incremented both in difficulty and complexity. Each module focused on either origami tasks or CAD tasks. 
Origami instructions incorporate numerous multi-step transformations of a square piece of paper that, if manipulated correctly, lead to a two or three dimensional finished product (Figures 1 and 2). Origami tasks were rated by the researchers on difficulty as defined by the number and types of folds as well as on the complexity of the task. Each origami module included basic symbol explanation, nomenclature, design instruction, and one to three origami tasks. Participants were required to physically fold a square piece of paper into the origami model by following the provided instructions.

CAD software allows users to create 3D drawings from given orthographic drawings (Figures 3 and 4). CAD tasks were rated in difficulty, by the researchers, depending on the types of corners as well as the number of layers and hidden lines of the object, and the intricacy of the design. The free online software SketchUp was utilized by workshop participants to create the 3D CAD drawings. Each CAD module contained basic design instructions and notation, a link to the SketchUp website, one example, and one to three tasks. In each task, participants were asked to create a CAD drawing of the object from the given multiview 2D flat images of the object's top, front, and side (which is the standard used in engineering and technology). Participants were also provided solutions for the previous module.

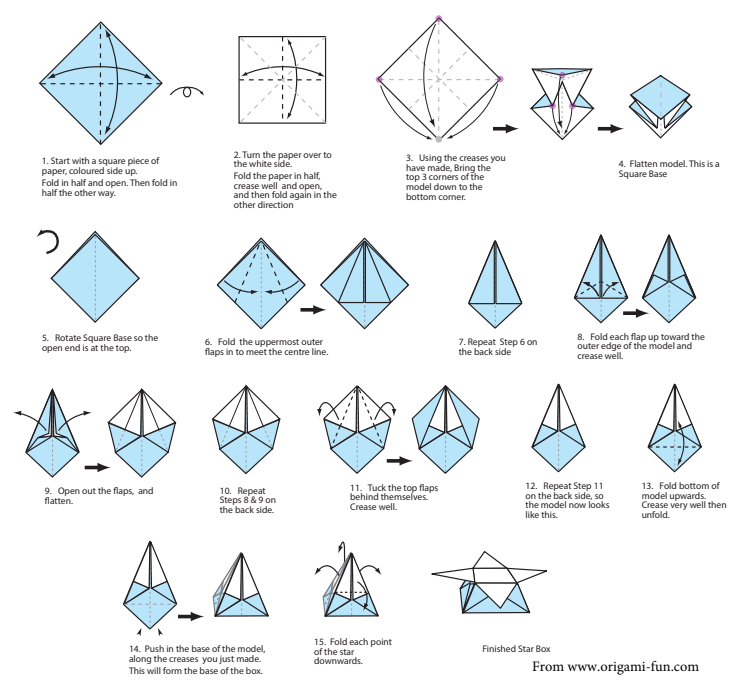

Figure 1: Example of origami task (O-LI-2) folding instructions ${ }^{19}$.

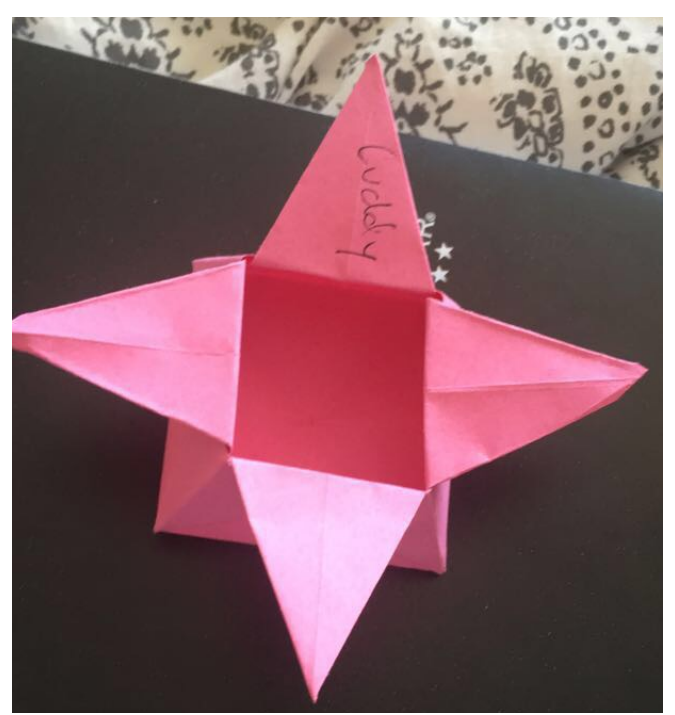

Figure 2: Deliverable of origami task $(\mathrm{O}-$ LI-2).

Modules were provided online via the course management system. Participants had one week to complete each module and submit the appropriate task deliverable(s) via the online system. The deliverable for each origami-based module was a photograph of the object(s) they created (Figure 2). The deliverable for each CAD-based module was a SketchUp file of their final drawing(s) (Figure 4). 


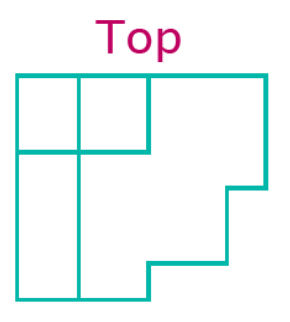

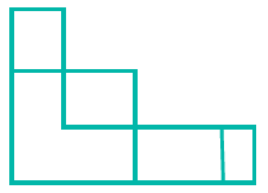

Front

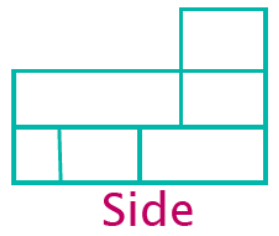

Figure 3: Example of CAD task (C-LI-1) multiview orthographic drawings instructions.

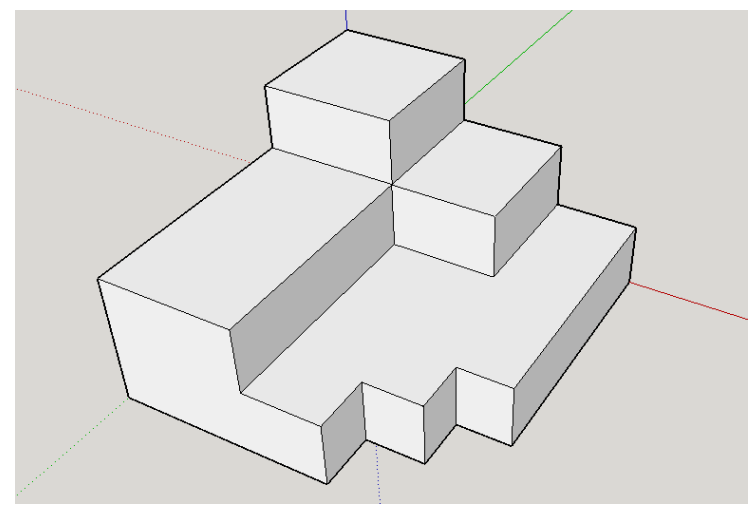

Figure 4: Deliverable of CAD task (C-LI-1).

\section{Methods}

\section{Research Questions}

This research is guided by the following research questions:

1. Does performing origami-based tasks and/or orthographic projections-based tasks positively impact spatial perception, mental rotation, and/or spatial visualization abilities?

If so:

- What proportion of origami/CAD activities generates the greatest increase in PSVT scores?

- What proportion of origami/CAD activities provides a quicker increase in PSVT scores?

2. Does the level of previous experience in origami/CAD impact PSVT scores?

\section{Workshop Design}

Four 12-week workshops utilizing origami and/or CAD exercises were developed. Workshop A consisted of four origami modules followed by eight CAD modules; workshop B is the reverse of A and consisted of the eight $\mathrm{CAD}$ modules followed by the four origami modules; workshop $\mathrm{C}$ contained 12 origami modules; workshop D had 12 CAD modules. The first module of each workshop highlighted the uses of origami and CAD in engineering, to emphasize the applicably of these tasks to their desired major.

Each module included multiple origami tasks or multiple CAD tasks. Each task was rated on the following difficulty scale: Simple (S - Figures 5(a) and 6(a)), Low Intermediate (LI - Figures 5(b) and 6(b)), Intermediate (I - Figures 5(c) and 6(c)), High Intermediate (HI - Figures 5(d) and 6(d)), Complex (C - Figures 5(e) and 6(e)). Each module became more difficult and complex than the 
previous. Modules with the same difficulty rating were then ordered with regard to complexity (Table 1).

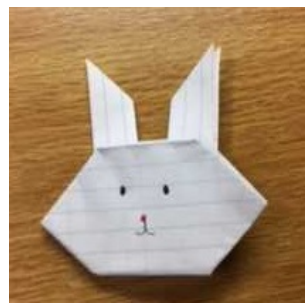

(a)

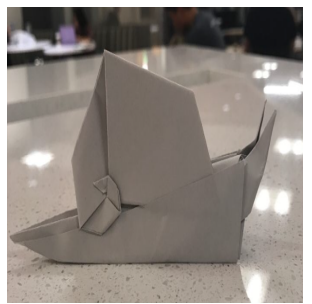

(b)

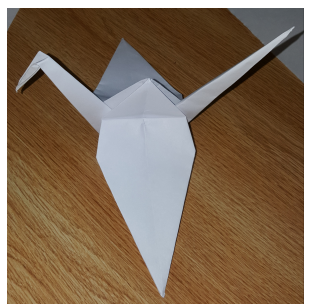

(c)

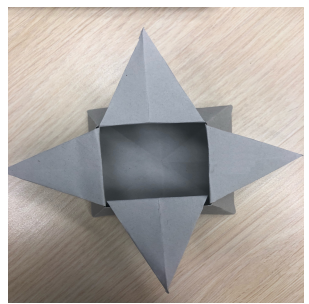

(d)

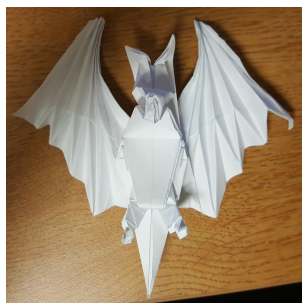

(e)

Figure 5: Origami examples of difficulty scale: (a) Simple, (b) Low Intermediate, (c) Intermediate, (d) High Intermediate, and (e) Complex

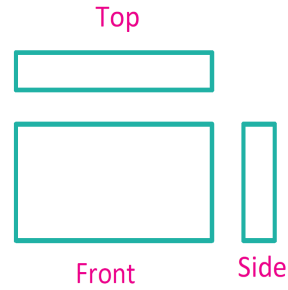

(a)

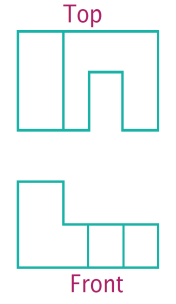

(b)

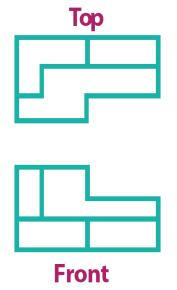

(c)

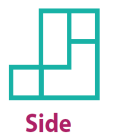

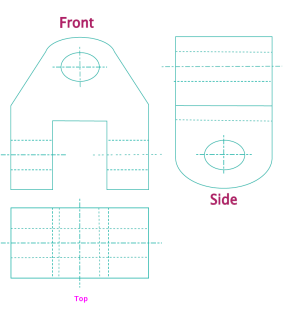

(d)

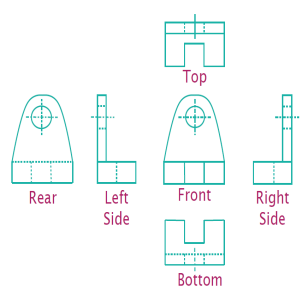

(e)

Figure 6: CAD examples of difficulty scale: (a) Simple, (b) Low Intermediate, (c) Intermediate, (d) High Intermediate, and (e) Complex

Table 1: Modules 1 through 12 for Workshops A - D. Module nomenclature: Type (O: origami or C: CAD) - Difficulty (S: Simple, LI: Low Intermediate, I: Intermediate, HI: High Intermediate, C: Complex) - Complexity (leveled 1 - 4)

\begin{tabular}{ccccc}
\hline Module & Workshop A & Workshop B & Workshop C & Workshop D \\
\hline 1 & O-S-1 & C-S-1 & O-S-1 & C-S-1 \\
2 & O-LI-1 & C-S-2 & O-S-2 & C-S-2 \\
3 & O-I-1 & C-S-3 & O-S-3 & C-S-3 \\
4 & O-C-2 & C-LI-1 & O-LI-1 & C-S-4 \\
5 & C-S-1 & C-LI-2 & O-LI-2 & C-LI-1 \\
6 & C-S-2 & C-I-2 & O-I-1 & C-LI-2 \\
7 & C-S-3 & C-HI-2 & O-I-2 & C-I-1 \\
8 & C-LI-1 & C-C-2 & O-I-3 & C-I-2 \\
9 & C-LI-2 & O-S-1 & O-HI-1 & C-HI-1 \\
10 & C-I-2 & O-LI-1 & O-HI-2 & C-HI-2 \\
11 & C-HI-2 & O-I-1 & O-C-1 & C-C-1 \\
12 & C-C-2 & O-C-2 & O-C-2 & C-C-2 \\
\hline
\end{tabular}




\section{Data Collection and Assessment Instrument}

The participants' spatial ability was assessed before starting the online workshop modules (pre-test), after the completion of the first four modules (mid-test), and after completion of all 12 modules (post-test). The assessment instrument used was the Purdue Spatial Visualization Test (PSVT), which consists of 90 questions and is divided into three sections of 30 questions each: (1) Rotations, (2) Views, and (3) Developments ${ }^{20}$.

The rotations section (PSVT:R) assesses subject's ability to mentally visualize rotations of 3D objects $^{21}$. Subjects are presented with an original object (object 1) and a view of the same object after rotation, then participants are asked to select the correct rotated view of the new object (object 2) that undergoes the same rotation as object 1 (refer to Figure $7(a))^{20}$. Participants had 12 minutes to answer the 30 questions $^{21}$.

The views section (PSVT:V) is designed to assess ones ability to determine the view of an object from different perspectives ${ }^{21}$. To this end, an object is set in an imaginary glass box and the participant is asked to select the correct representation from a specific view point (refer to Figure $7(\mathrm{~b}))^{20}$. Participants were allowed 20 minutes to answer the 30 items in this section ${ }^{21}$.

The developments (PSVT:D) section is divided into two sub-sections of 15 questions each ${ }^{20}$. The first sub-section assesses how well the subject can imagine the folding of a $2 \mathrm{D}$ object into a 3D object (refer to Figure 7(c)) ${ }^{21}$. The second sub-section asks participants to determine the 2D view of an unfolded 3D object (refer to Figure 7(d) $)^{21}$. Participants were allowed a total of 18 minutes (nine minutes for each sub-section) for this section ${ }^{21}$.

Assessments were administered through an online portal. The virtual tool allowed participants to select only one answer for each item, move forward and backward, review answers, and included a time and progress bar for reference. The assessments closed automatically after the allotted time frame.

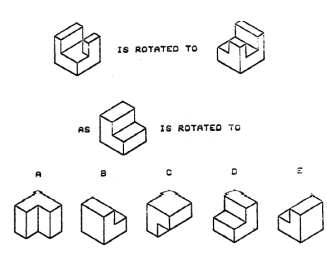

(a)

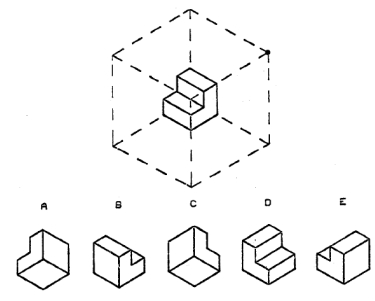

(b)

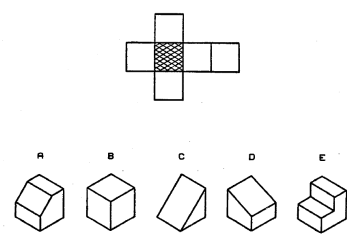

(c)

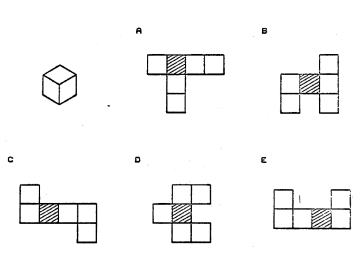

(d)

Figure 7: Sample questions of (a) PSVT:R, (b) PSVT:V, (c) PSVT:D sub-section 1 and (d) PSVT:D sub-section 2 (the correct answers are $D, E, B$ and $C$ respectively) ${ }^{16}$.

\section{Participants}

The study sample was comprised of 211 female students enrolled in a first-year engineer seminar course that focuses on encouraging success. The course is offered each Fall semester and enrolls predominantly female first-year engineering students. All students were required to complete the 
PSVT surveys and randomly assigned to a treatment (control, A, B, C, or D) as part of the required class work.

\section{Demographics}

Demographics were collected on residency, parent/guardian careers, and experience in origami and CAD. Table 2 further delineates the demographics of the participants in each workshop group and the control group.

In the control group, $40.74 \%$ (22) of the participants were in-state (i.e. from the state where the institution is located), $53.70 \%$ (29) were from another U.S. state, and 5.56\% (3) international. The experimental group had $40.13 \%$ (63) from Indiana, 56.69\% (89) from other U.S. states, and 3.18\% (5) international participants.

Table 2: Control and experimental groups' self-reported residency, number of parents/guardians who are engineers, and experience in origami and CAD prior to workshop

\begin{tabular}{lccccc} 
Demographic & Control & $\mathrm{A}$ & $\mathrm{B}$ & $\mathrm{C}$ & $\mathrm{D}$ \\
\hline In-state & 22 & 16 & 13 & 14 & 20 \\
Other U.S. Domestic & 29 & 19 & 23 & 29 & 18 \\
International & 3 & 1 & 1 & 2 & 1 \\
& & & & & \\
No parents engineers & N/A & 28 & 26 & 26 & 26 \\
One parent engineer & N/A & 6 & 7 & 15 & 10 \\
Multiple parents engineers & N/A & 2 & 4 & 4 & 3 \\
& & & & & \\
Origami: None & N/A & 18 & 15 & 27 & 24 \\
Origami: Beginner & N/A & 16 & 18 & 15 & 14 \\
Origami: Intermediate & N/A & 2 & 4 & 3 & 1 \\
& & & & & \\
CAD: None & N/A & 17 & 14 & 26 & 19 \\
CAD: Beginner & N/A & 11 & 6 & 6 & 11 \\
CAD: Intermediate & N/A & 6 & 13 & 10 & 5 \\
CAD: Advanced & N/A & 2 & 4 & 3 & 4 \\
\hline Total per Group & 54 & 36 & 37 & 45 & 39 \\
\hline
\end{tabular}

Only participants in the experimental group were asked to report on the status of their parents/guardians as engineers. Thirteen $(8.28 \%)$ of the participants reported that they had multiple parents/guardians that were engineers, $24.20 \%$ (38) reported that one parent/guardian was an engineer, and $67.52 \%$ (106) had no parents/guardians as engineers.

Participant's in the experimental group also self-reported on their level of experience in both origami and CAD. The defined levels of experience are as follows:

- Origami Experience Levels:

- None

- Beginner (know how to read diagrams, know how to do basic folds such as mountain and valley, complete figures up to 20 steps)

- Intermediate (complete figures up to 40 steps, explore new techniques of origami)

- Advanced (complete figures with more than 40 steps, perform circular packing and tiles) 
- CAD Experience Levels:

- None

- Beginner (opening files, changing colors, drawing lines)

- Intermediate (import figures, draw 2D sketches, add text, add scale)

- Advanced (draw 3D sketches, do revisions, use dynamic menu)

Regarding origami, $53.50 \%$ (84) of the participants reported no experience, $40.13 \%$ (63) of the participants reported beginner experience, $6.37 \%$ (10) of the participants reported intermediate experience, and none reported advanced experience. Regarding CAD, $48.41 \%$ (76) of the participants reported no experience, $21.66 \%$ (34) of the participants reported beginner experience, $21.66 \%$ (34) of the participants reported intermediate experience, and $8.28 \%$ (13) reported advanced experience.

\section{Scoring and Data Analysis}

The data used in this analysis were the participants' results from the pre, mid, and post PSVTs. Participants' responses were assigned one point for each correct answer and no points for an incorrect response. Answers were scored automatically by the surveying tool. A manual systematic review of the data was conducted and inconsistent data were excluded from both the control and experimental sample pools. Data were labeled as inconsistent if any one PSVT subtest was not completed, or if an earned score was below 10 and the time used to complete the survey was less than or equal to one-third of the allotted time. Additionally, only data from first semester, first-year female engineering students were considered for this study. After removing inconsistent data, there were a total of 54 students in the control group, and 36, 37, 45 and 39 students in workshop groups $\mathrm{A}, \mathrm{B}, \mathrm{C}$ and $\mathrm{D}$ respectively. The research team is confident in the validity of the remaining data set.

The data analysis was conducted to examine workshop impact on spatial ability, the differences between treatments, and the influence of workshop content. Both descriptive and inferential statistics were employed. In the descriptive stage the mean, standard deviation, minimum, maximum, and confidence intervals from the pre-test, mid-test, and post-test for each treatment were obtained. The inferential stage consisted of a folded F statistic, using a 0.05 confidence level, conducted on the pre-test scores to determine if the experimental and control groups were comparable. A nested factorial analysis was conducted to determine if the treatments were statistically different. The following model (Equation 1) was used:

$$
S_{i j k l}=\mu+T_{i}+I_{(i) j}+A_{k}+T A_{i k}+A I_{(i) j k}+\varepsilon_{l(i j k)}
$$

where:

$$
\begin{aligned}
& \mu=\text { mean } \\
& \mathbf{I}=\text { subjects } \\
& \mathbf{T}=\text { treatments applied (control, A, B, C, and D) } \\
& \mathbf{A}=\text { each data collection (pre-test, mid-test, and post-test) } \\
& \mathbf{S}=\text { score on the tests } \\
& \varepsilon=\text { error }
\end{aligned}
$$


Tukey HSD was applied to any statistically significant source of variability in the model. Finally, a n-way ANOVA was conducted to compare pre-test PSVT scores using the origami and CAD levels to determine the impact of previous experience.

\section{Results}

\section{Description of the data set}

The control group earned the lowest mean score (16.85) among the five treatments on the PSVT:V pre-test while participants in the D workshop group obtained the highest mean score (20.10). On the mid-test, the control group continued to rank lowest on mean score with 20.11, while treatment $\mathrm{B}$ ranked highest with 22.68. On the post-test, the control group again scored the lowest (21.04), and treatment $\mathrm{D}$ ranked highest (23.56). Table 3 provides means, maximum and minimum values, standard deviations, and lower and upper confidence intervals for the PSVT:V pre-test, mid-test, and post-test for each treatment.

Table 3: Descriptive statistics of PSVT:V

\begin{tabular}{llllllll}
\hline Assessment & Treatment & Min & Max & Mean & Std Dev & Lower 95\% CL & Upper 95\% CL \\
\hline \multirow{5}{*}{ Pre-test } & Control & 4 & 28 & 16.8518 & 6.8443 & 14.9836 & 18.7200 \\
& A & 4 & 30 & 19.3055 & 6.5935 & 17.0746 & 21.5365 \\
& B & 2 & 29 & 18.2702 & 6.8176 & 15.9971 & 20.5433 \\
& C & 5 & 29 & 19.6000 & 6.9098 & 21.6759 & 19.6000 \\
& D & 7 & 30 & 20.1025 & 6.1763 & 18.1004 & 22.1025 \\
& & & & & & & \\
& Control & 9 & 30 & 20.1111 & 5.8557 & 18.5128 & 21.7094 \\
& A & 11 & 30 & 22.1388 & 6.1139 & 20.0703 & 24.2075 \\
Mid-test & B & 7 & 30 & 22.6756 & 6.1646 & 20.6202 & 24.7310 \\
& C & 7 & 30 & 21.6666 & 6.7588 & 19.6360 & 23.6972 \\
& D & 6 & 30 & 22.4615 & 6.2147 & 20.4469 & 24.4761 \\
& & & & & & & \\
& Control & 8 & 29 & 21.0370 & 6.3956 & 19.2913 & 22.7827 \\
& A & 11 & 30 & 23.3611 & 5.5915 & 21.4691 & 25.2530 \\
& B & 7 & 30 & 23.3783 & 5.7655 & 21.4560 & 25.3007 \\
& C & 10 & 30 & 23.2000 & 6.1923 & 21.3396 & 25.0603 \\
& D & 8 & 30 & 23.5641 & 6.0515 & 21.6024 & 25.5257 \\
\hline
\end{tabular}

On the PSVT:R, the control group obtained the lowest mean score on the pre-test (16.65), mid-test (18.02), and post-test (18.67). On the other hand, the highest ranking treatment for the pre-test (19.11) and mid-test (20.73) was C while B (22.00) earned the highest score on the post-test. Table 4 provides the descriptive statistics obtained for PSVT:R.

Table 5 shows that the control group ranked lowest on the pre, mid, and post PSVT:D tests with scores of 16.80, 19.30, and 21.85 respectively. Treatment B participants achieved the highest mean scores for the pre-test with 19.41, mid-test with 22.46, and 25.15 for the post-test. 
Table 4: Descriptive statistics of PSVT:R

\begin{tabular}{clllllll}
\hline Assessment & Treatment & Min & Max & Mean & Std Dev & Lower 95\% CL & Upper 95\% CL \\
\hline \multirow{5}{*}{ Pre-test } & Control & 8 & 28 & 16.6481 & 4.6099 & 15.3898 & 17.9064 \\
& A & 11 & 28 & 18.3888 & 3.8342 & 17.0915 & 19.6862 \\
& B & 10 & 25 & 18.6486 & 4.3856 & 17.1863 & 20.1109 \\
& C & 7 & 30 & 19.1111 & 5.4365 & 17.4778 & 20.7444 \\
& D & 7 & 27 & 17.9487 & 4.9944 & 16.3297 & 19.5677 \\
& & & & & & & \\
Mid-test & Control & 9 & 28 & 18.0185 & 4.6923 & 16.7377 & 19.2992 \\
& A & 12 & 27 & 20.6667 & 4.2961 & 19.2130 & 22.1202 \\
& B & 8 & 28 & 19.7297 & 4.8456 & 18.1141 & 21.3453 \\
& C & 6 & 29 & 20.7333 & 5.7894 & 18.9939 & 22.4726 \\
& D & 6 & 30 & 20.6667 & 5.0644 & 19.0249 & 22.3083 \\
& & & & & & & \\
& Control & 7 & 28 & 18.6667 & 5.0767 & 17.2809 & 20.0523 \\
& A & 12 & 30 & 21.4167 & 4.4809 & 19.9005 & 22.9327 \\
& B & 8 & 30 & 22.0000 & 4.7258 & 20.42433 & 23.5756 \\
& C & 10 & 30 & 21.4889 & 5.3113 & 19.8931 & 23.0845 \\
& D & 8 & 30 & 20.9487 & 5.7808 & 19.0747 & 22.8226 \\
\hline
\end{tabular}

Table 5: Descriptive statistics of PSVT:D

\begin{tabular}{clllllll}
\hline Assessment & Treatment & Min & Max & Mean & Std Dev & Lower 95\% CL & Upper 95\% CL \\
\hline \multirow{5}{*}{ Pre-test } & Control & 8 & 27 & 16.7962 & 4.8423 & 15.4745 & 18.1180 \\
& A & 6 & 28 & 17.4444 & 4.6687 & 15.8647 & 19.0241 \\
& B & 9 & 30 & 19.4054 & 5.4490 & 17.5886 & 21.2222 \\
& C & 7 & 27 & 17.3333 & 5.2005 & 15.7709 & 18.8957 \\
& D & 8 & 29 & 18.4615 & 4.5759 & 16.9781 & 19.9448 \\
& & & & & & & \\
Mid-test & Control & 8 & 29 & 19.2962 & 4.8423 & 15.4745 & 18.1180 \\
& A & 9 & 29 & 20.9722 & 4.6687 & 15.8647 & 19.0241 \\
& B & 14 & 30 & 22.4594 & 5.4490 & 17.5886 & 21.2222 \\
& C & 6 & 30 & 20.9333 & 5.2005 & 15.7709 & 18.8957 \\
& D & 9 & 30 & 21.3076 & 4.5759 & 16.9781 & 19.9448 \\
& & & & & & & \\
& Control & 11 & 30 & 21.8464 & 5.0000 & 19.1164 & 21.8464 \\
& A & 11 & 29 & 23.5463 & 4.9808 & 20.1758 & 23.5463 \\
& B & 9 & 30 & 25.1461 & 4.5725 & 22.0970 & 25.1461 \\
& C & 10 & 30 & 23.5826 & 5.6376 & 20.1951 & 23.5826 \\
& D & 14 & 29 & 24.5085 & 4.0208 & 21.9017 & 24.5085 \\
\hline
\end{tabular}


Are the treatments comparable?

An one-way ANOVA conducted on the pre-test scores of PSVT:V, PSVT:R, and PSVT:D determined that the treatments are not statistically different, thus allowing comparison of the treatment scores from the mid-tests and post-tests. Table 6 summarizes the results of this analysis.

Table 6: One-way ANOVA for PSVT:V, PSVT:R, and PSVT:D.

\begin{tabular}{llll} 
& Source & F Ratio & p-value \\
\hline PSVT:V & Model & 1.77 & 0.1356 \\
PSVT:R & Model & 1.96 & 0.1016 \\
PSVT:D & Model & 1.84 & 0.1218 \\
\hline
\end{tabular}

$\left.{ }^{*}\right)$ indicates that the $\mathrm{p}$-value $\leq 0.05$.

\section{Comparison of treatments}

The nested model analysis of the PSVT:V data gave an $R^{2}$ of 0.854 which indicates that approximately $85 \%$ of the total variation in the scores can be explain by assessment repetitions, treatments, and the interaction of the treatments and assessments repetitions. There is a statistically significant difference, p-value less than 0.0001, in the PSVT:V scores between assessment repetitions (Table 7). Hence, there is a significant difference between pre, mid and post tests. A Tukey HSD was run to determine which tests are different from the others. The analysis of the Tukey HSD showed that the scores could be divided in two groups: (1) Pre-score of control, A, B, C, D, and mid-score C and (2) mid-score of control, A, B, D and post-scores of control, A, B, C, and D. Thus, all treatments significantly changed their mean score by the mid-test except for C. For control, A, B, and D the change showed in the post-test was not significantly different from the change showed after the first four weeks. In the case of treatment $\mathrm{C}$, a significant change was only observed after 12 weeks. A graphical representation of the treatments behavior can be found in Figure 8(a).

In the analysis of PSVT:R scores using the nested model an $R^{2}$ of 0.771 was obtained, which signaled that approximately $77 \%$ of the variance in the scores could be explained by the model. The fixed effect test showed that treatment and assessment repetition means were significantly different (refer to Table 7). The control group did not show a significant difference in their scores after four or 12 weeks. Participants in workshops A, C, and D significantly increased their scores after four weeks, however their scores did not significantly increase after the additional eight weeks.

Treatment B did not show a significant change after four modules, but the increase was significant after 12 modules. Post-test scores of A, B, C and D were statistically different from the score of the control, however, they were similar between each other. Figure $8(\mathrm{~b})$ portrays the treatments mean scores at each repetition.

The PSVT:D $R^{2}$ of 0.815 suggests that approximately $81 \%$ of the score variations can be explain by the model. The analysis shows that assessment repetitions (p-value less than 0.0001) and treatments $(p$-value $=0.0299)$ are significantly different. However, the interaction of these variables is not 
significantly different (refer to Table 7). The control group scores did not change significantly after four or 12 weeks. Mean scores changed significantly after four weeks for all experimental treatments, however the eight additional modules did not significantly alter the post-test mean scores (refer to Figure 8(c) to observe the results).

Table 7: Nested Factorial Analysis for PSVT scores

\begin{tabular}{llll} 
& Source & F Ratio & p-value \\
\hline \multirow{3}{*}{ PSVT:V } & Treatment & 1.6459 & 0.1640 \\
& Test & 99.3738 & $<0.0001^{*}$ \\
& Test x Treatment & 0.9631 & 0.4644 \\
& & & \\
PSVT:R & Treatment & 3.1416 & $0.0155^{*}$ \\
& Test & 47.2597 & $<0.0001^{*}$ \\
& Test x Treatment & 1.0405 & 0.4046 \\
& & & \\
PSVT:D & Treatment & 2.7377 & $0.0299 *$ \\
& Test & 131.9266 & $<0.0001^{*}$ \\
& Test x Treatment & 0.5178 & 0.8432 \\
\hline
\end{tabular}

$\left({ }^{*}\right)$ indicates that the $\mathrm{p}$-value $\leq 0.05$.

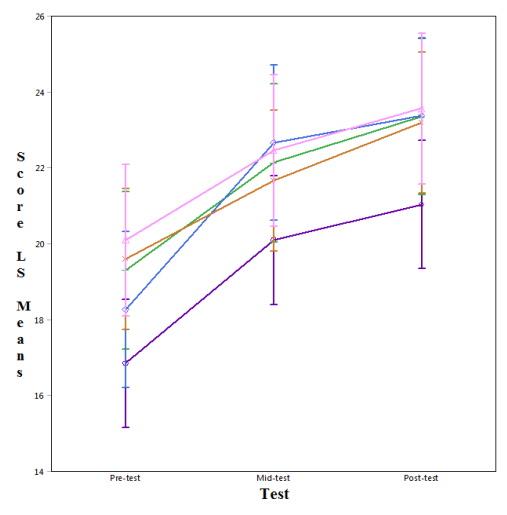

(a)

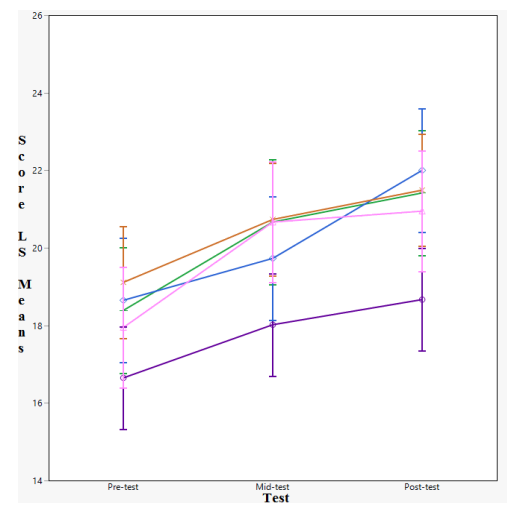

(b)

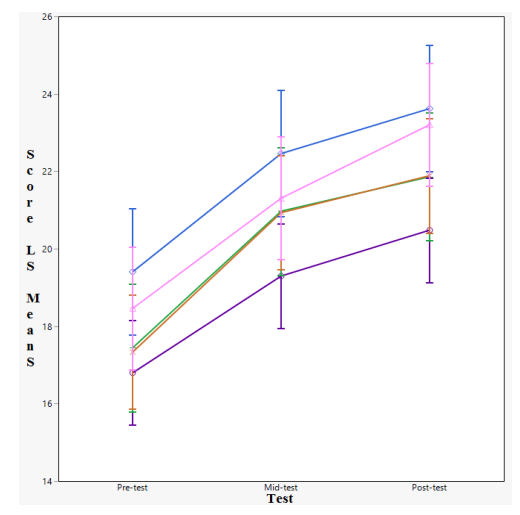

(c)

Figure 8: Treatments vs. assessments LS Means Plot of (a) PSVT:V scores, (b) PSVT:R scores, and (c) PSVT:D scores. Control: $\odot$ A: + , B: $\diamond-, C:$ , and D:

\section{Do demographics matter?}

The experimental groups' PSVT pre-test scores were analyzed using a n-way ANOVA test in the context of demographic information: residency, the number of parents/guardians that are engineers, and their origami and CAD experience. An alpha of 0.05 was used to determine significance.

In-state participants earned the highest scores on the PSVT:V, followed by U.S. Domestic, then International participants ( $\mathrm{p}$-value $=0.018$ ). Participants having more than one engineering 
parent/guardian performed worse than participants with only one or no engineering parent/guardian on both the PSVT:V (p-value $=0.005)$ and PSVT:R $(\mathrm{p}=0.010)$.

Participants with Beginner origami experience performed better than those with none on the PSVT:V (p-value $=0.046)$ and PSVT:R $(p$-value $=0.033)$. Prior origami experience may also lead to better performance on the PSVT:D (p-value $=0.077)$.

Participants with Intermediate or Advanced CAD experience performed better on the PSVT:R ( $\mathrm{p}$-value $=0.043$ ) compared to those that reported Beginner or no experience.

\section{Discussion}

The goal of this work was to develop a readily accessible training tool for first-year female engineering students to quickly improve their spatial abilities. Participants in workshops A and B completed a combination of origami and CAD, while those in workshops $\mathrm{C}$ and $\mathrm{D}$ completed only one type of training.

Participants in the control, A, B, and D treatments showed significant improvement of their PSVT:V scores after only four weeks whereas participants in the $\mathrm{C}$ workshop only saw significant improvement after all 12 modules. Results suggest that the use of simple origami tasks somehow retard the development of spatial perception skills. Participants in treatment D obtained the highest mean score by the mid-test, while participants in B scored highest after 12 weeks. Both workshops $\mathrm{D}$ and $\mathrm{B}$ employed CAD tasks at the beginning of their training, however, it seems that the inclusion of origami tasks after CAD may help students. This may be the result of participants having to practice imagining an object from different perspectives as was necessary to complete the CAD modules. These results aligned with the ones obtained by Sorby ${ }^{22}$ and Shavalier ${ }^{23}$, who previously studied the effect of using CAD software.

All experimental treatments had a positive statistically significant increase on the PSVT:R after 12 weeks. However, A, C, and D only required four weeks to achieve a significant difference, while participants in B took 12 weeks. Additionally, after the 12 weeks, workshop B participants earned the highest mean score. These results suggest that the quickest way to increase mental rotation skills would be to use either origami tasks or simple CAD tasks. Sorby ${ }^{2}$ concluded that the use of sketching benefited the development of spatial skills. Our findings show that sketching using CAD software also significantly impacts mental rotation skills. Yue and $\mathrm{Chen}^{24}$ concluded that the use of CAD did not impact spatial skills, however, our findings show that the correct CAD task sequencing or more time allowed for training positively impacts spatial ability. Additionally, our results using origami contradict Boakes ${ }^{11}$ findings, who used origami activities for teaching mathematics and geometry to middle school students.

The control treatment did not significantly change scores after either four or 12 weeks on the PSVT:D. On the other hand, all experimental treatments achieved positive significant change by four weeks. The two treatments that employed CAD at the beginning of the training earned the highest mean scores. These results suggest that four weeks are sufficient to increase visualization skills using either CAD or origami tasks. Our results agree with Khine ${ }^{25}$, who expressed that origami based instructions have a positive impact on visualization skills. 
Students with Intermediate or Advanced CAD experience scored higher on the PSVT:R than those with no CAD experience. A 1999 study by Field ${ }^{26}$ noted that using CAD software may not contribute to spatial skills; however, in 2014 Cohen $^{27}$ found that CAD drawings using animations have been shown to be effective at increasing spatial skills in a short-term training experience. The benefits of using CAD to improve spatial abilities are still being explored however, we were expecting that students with any level of CAD experience would earn higher pre-test scores on all three PSVTs than those with no CAD experience.

Participants that noted Beginner experience with origami scored higher on all three of the PSVT pre-tests than those with no reported experience. Prior research noted above indicates that origami helps train grade and middle school students in spatial visualization. These results indicate that origami also improves ones' spatial perception and mental rotation skills as well.

\section{Implications for implementation}

Those wishing to implement a similar workshop should note that the individual tasks in the origami and CAD modules may be recreated and/or replaced with similar objects; however, assessment of student improvement is recommended. Also note that detailed instructions (either written or visual such as an online tutorial) are needed for students to complete tasks without supervision. Finally, if modules are implemented online, a reliable avenue to collect deliverables must be established.

\section{Conclusions}

This research shows that after just four weeks of an online training workshop, participants significantly increase their spatial ability aptitude. Spatial perception can be increased using only CAD activities or origami tasks that increase in complexity. Mental rotation can be increased significantly using only simple origami tasks, origami tasks that increase in complexity, or simple CAD tasks. Spatial visualization skills can also be significantly increased with origami or CAD tasks, regardless of complexity. Even though significant increases in spatial ability can be achieved after four weeks using any of the four designed workshops, treatment B (eight CAD modules followed by four origami modules) outperforms all other treatments for improving spatial perception, mental rotation, and spatial visualization skills.

\section{Future Work}

Impact of task complexity will be examined in future experiments. Training and testing of male engineering students will be carried out for workshop validation. Additionally, sustained increase of spatial skills could also be studied by the implementation of a longitudinal study. 


\section{References}

[1] D. H. Uttal, D. I. Miller, and N. S. Newcombe, "Exploring and enhancing spatial thinking: Links to achievement in science, technology, engineering, and mathematics," Current Directions in Psychological Science, vol. 22, no. 5, pp. 367-373, 2013.

[2] S. A. Sorby, "Educational research in developing 3-D spatial skills for engineering students," International Journal of Science Education, vol. 31, no. 3, pp. 459-480, 2009.

[3] F. T. Siemankowski and F. C. MacKnight, "Spatial cognition, a success prognosticator in college science courses," in Proceedings of the 44th Annual Meeting of the National Association for Research in Science Teaching, Silver Spring, MD, 1971.

[4] S. S. Metz, S. A. Sorby, and T. Jarosewich, "Spatial skills training impacts retention of engineering students: Does this success translate to community college students in technical education?" in Proceedings of the American Society for Engineering Education Annual Conference and Exposition, New Orleans, LA, 2016.

[5] N. S. Newcombe, "Picture this: Increasing math and science learning by improving spatial thinking," American Educator, vol. 34, no. 2, pp. 29-43, 2010.

[6] G. Park, D. Lubinski, and C. P. Benbow, "Recognizing spatial intelligence: Our schools, and our society, must do more to recognize spatial reasoning, a key kind of intelligence," National Geographic. Retrieved from http://www. scientificamerican. com/article. cfm, Nov. 2010.

[7] C. A. Cohen and M. Hegarty, "Inferring cross sections of 3-D objects: A new spatial thinking test," Learning and Individual Differences, vol. 22, no. 6, pp. 868-874, 2012.

[8] J. R. Power, S. A. Sorby, N. L. Veurink, K. Atit, and M. Carr, "Preparing students for engineering success through improving 3-D spatial skills," in Proceedings of the American Society of Engineering Education Annual Conference and Exposition, Columbus, OH, 2017.

[9] G. Duffy, S. A. Sorby, A. Mack, and B. Bowe, "Performance by gender on university placement tests in mathematics and spatial skills," in Proceedings of the American Society for Engineering Education Annual Conference and Exposition, Columbus, OH, 2017.

[10] D. H. Uttal, N. G. Meadow, E. Tipton, L. L. Hand, A. R. Alden, C. Warren, and N. S. Newcombe, "The malleability of spatial skills: A meta-analysis of training studies," American Psychological Association, vol. 139, no. 2, pp. 352-402, 2013.

[11] N. J. Boakes, "Origami instruction in the middle school mathematics classroom: Its impact on spatial visualization and geometry knowledge of students," Research in Middle Level Education Online, vol. 32, no. 7, pp. 1-12, 2009.

[12] S. Cakmak, M. Isiksal, and Y. Koc, "Investigating effects of origami-based instruction on elementary students' spatial skills and perceptions," The Journal of Educational Research, vol. 107, no. 1, pp. 59-68, 2014.

[13] V. Toptas, S. Celik, and E. Karaca, "Improving 8th graders spatial thinking abilities through a 
3d modeling program," Turkish Online Journal of Educational Technology, vol. 11, no. 2, pp. 128-134, 2012.

[14] B. Guven and T. Kosa, "The effect of dynamic geometry software on student mathematics teachers' spatial visualization skills," Turkish Online Journal of Educational Technology, vol. 7, no. 4, pp. 100-107, 2008.

[15] S. Hsi, M. C. Linn, and J. E. Bell, "The role of spatial reasoning in engineering and the design of spatial instruction," Journal of Engineering Education, vol. 86, no. 2, pp. 151-158, 1997.

[16] S. Zurn-Birkhimer, M. Serrano, B. Holloway, and R. Baker, "Work in progress: Online training in spatial reasoning for first-year female engineering students," in Proceedings of the American Society of Engineering Education Annual Conference and Exposition, Salt Lake City, UT, 2018.

[17] Z. Xiao, Y. Yao, C.-H. Yen, S. Dey, H. Wauck, J. Leake, B. Woodard, A. Wolters, and W.-T. $\mathrm{Fu}$, "A scalable online platform for evaluating and training visuospatial skills of engineering students," in Proceedings of the American Society of Engineering Education Annual Conference and Exposition, Columbus, OH, 2017.

[18] M. Contero, F. Naya, P. Company, J. L. Saorin, and J. Conesa, "Improving visualization skills in engineering education," IEEE Computer Graphics and Applications, vol. 25, no. 5, pp. 24-31, 2005.

[19] R. Hondow. Origami-fun.com. [Online]. Available: https://www.origami-fun.com/support-files/origami-star-box-print.pdf

[20] R. Guay, Purdue Spatial Visualization Test. West Lafayette, IN: Purdue Research Foundation, 1976.

[21] J. Eliot and I. M. Smith, An international directory of spatial tests. Windsor, Berkshire: NFER-Nelson, 1983.

[22] S. A. Sorby, "Spatial abilities and their relationship to computer aided design instruction," in Proceedings of the American Society of Engineering Education Annual Conference and Exposition, Charlotte, NC, 1999.

[23] M. Shavalier, "The effects of CAD-like software on the spatial ability of middle school students," Journal of Educational Computing Research, vol. 31, no. 1, pp. 37-49, 2004.

[24] J. Yue and D. M. Chen, "Does CAD improve spatial visualization ability?" in Proceedings of the American Society of Engineering Education Annual Conference and Exposition, Albuquerque, NM, 2001.

[25] M. S. Khine, Visual-spatial Ability in STEM Education. Switzerland: Springer International Publishing, 2016.

[26] B. Field, “A course in spatial visualization,” Journal for Geometry and Graphics, vol. 3, no. 2, pp. 201-209, 1999.

[27] C. A. Cohen and M. Hegarty, "Visualizating cross sections: Training spatial thinking using 
interactive animations and virtual objects," Learning and Individual Differences, vol. 33, pp. 63-71, 2014. 